\title{
Multiuser MIMO-OFDM systems using subcarrier hopping
}

\author{
M. Jiang and L. Hanzo
}

\begin{abstract}
Recently space division multiple access (SDMA) assisted multiple-input-multiple-output (MIMO) orthogonal frequency division multiplexing (OFDM) systems invoking multiuser detection (MUD) techniques have attracted substantial research interest, which is capable of exploiting both transmitter multiplexing gain and receiver diversity gain. A new scheme referred to here as slowsubcarrierhopping (SSCH) assisted multiuser SDMA-OFDM, is proposed. It is shown that, with the aid of the so-called uniform SSCH (USSCH) pattern, the multiuser interference (MUI) experienced by the high-throughput SDMA-OFDM system can be effectively suppressed, resulting in a significant performance improvement. In the investigations conducted, the proposed USSCH-aided SDMA-OFDM system was capable of outperforming a range of SDMA-OFDM systems considered, including the conventional SDMA-OFDM system dispensing with the employment of frequency-hopping techniques. For example, at an $E_{b} / N_{0}$ value of $12 \mathrm{~dB}$, the proposed USSCH/SDMA-OFDM system reduced the bit error ratio (BER) by about three orders of magnitude, in comparison to the conventional SDMA-OFDM system, while maintaining a similar computational complexity.
\end{abstract}

\section{Introduction}

A range of time division multiple access (TDMA) [1], frequency division multiple access (FDMA) [1] and code division multiple access (CDMA) [2, 3] schemes have found employment in first-, second- and third-generation wireless systems. Spread-spectrum multiple access (SSMA) [4] schemes have been widely investigated since they exhibit a range of attractive properties, including the ability of combating various types of interference. A classic SSMA scheme is constituted by frequency-hopped SSMA (FH/ SSMA) [3, 5-8], where the total available system bandwidth is divided into a number of sub-bands shared by a number of users. FH can be effectively amalgamated with the well established technique of orthogonal frequency division multiplexing (OFDM) [9, 10], which exhibits a number of advantages over more traditional multiplexing techniques, resulting in $\mathrm{FH} / \mathrm{OFDM}$ systems [11]. Furthermore, space division multiple access (SDMA) based OFDM [10, 13] communication invoking multiuser detection (MUD) $[10,13]$ techniques has recently attracted intensive research interest. In SDMA multiple-input-multiple-output (MIMO) systems, the transmitted signals of $L$ simultaneous uplink mobile users, each equipped with a single transmit antenna, are received by the $P$ different receiver antennas of the base station (BS). At the BS the individual users' signals are separated with the aid of their unique, user-specific spatial signature constituted by their channel transfer functions or, equivalently, channel impulse responses (CIRs).

However, conventional SDMA-OFDM systems [10, 12], where all the users exploit the entire system bandwidth

(C) The Institution of Engineering and Technology 2006

IEE Proceedings online no. 20050412

doi:10.1049/ip-com:20050412

Paper first received 2nd August 2005 and in revised form 2nd March 2006

The authors are with the School of Electronics and Computer Science, University of Southampton, Salisbury Rd, Southampton SO17 1BJ, UK

E-mail: 1h@ecs.soton.ac.uk for their communications, exhibit a few drawbacks. The conventional SDMA-OFDM systems can exploit little frequency diversity, since each user activates all available subcarriers. This limitation can be mitigated by combining both slow frequency-hopping (SFH) and SDMA-OFDM techniques, resulting in the SFH/SDMA-OFDM systems. In SFH/SDMA-OFDM systems the total system bandwidth is divided into several sub-bands, each of which hosts a number of consecutive subcarriers, and a so-called SFH pattern is used for controlling the sub-band allocation for the different users. Since each user activates different subbands from time to time, the achievable frequency diversity improves, as the width of the sub-bands is reduced.

However, when the number of users becomes higher in conventional SDMA-OFDM systems, a higher multiuser interference (MUI) is expected across the entire bandwidth, and hence all users will suffer from a performance degradation. Unfortunately, the same phenomenon is encountered also in SFH/SDMA-OFDM systems at those sub-bands that are shared by an excessive number of users. Undoubtedly, the best solution to eliminate the MUI is to avoid sub-band collisions between the different users by assigning each sub-band exclusively to a single user. This 'one-subband-for-one-user' scheme will inevitably reduce the system's overall throughput. The attainable system throughput can be increased with the aid of higher-order modems, which are more vulnerable to transmission errors and also impose an increased MUD complexity at the receivers, which is undesirable. Therefore, subcarrier reuse based SDMA-OFDM using efficient frequency-hopping techniques is preferable, since it is capable of maintaining a sufficiently high overall system throughput even with the employment of a relatively low-order, low-complexity modem, while effectively suppressing the associated high MUI.

Against this background, the novel contribution of this paper is that the performance of conventional multiuser SDMA-OFDM systems is significantly improved by employing a novel subcarrier-based SFH technique, 
referred to here as the slow-subcarrierhopping ( $\mathrm{SSCH}$ ) scheme. More specifically, using a simple but efficient uniform SSCH (USSCH) pattern, the SSCH/SDMAOFDM system is capable of achieving a high frequency diversity, and hence exhibits a high robustness to MUI. In the literature, substantial research efforts have been invested in designing subcarrier allocation algorithms for singleinput-single-output (SISO) OFDM systems, which are subject to various design constraints, such as requiring the minimal overall transmit power [14-19], achieving the maximum capacity [19-21] or complying with specific quality-of-service (QoS) criteria [22], etc. A subcarrier and bit allocation algorithm designed for minimising the overall transmit power for a MIMO-OFDM systems was proposed in [23]. Furthermore, a number of sub-band/subcarrier allocation schemes were also proposed for systems based on clustered OFDM (ClOFDM) [24] or orthogonal frequency division multiple access (OFDMA) [25-28]. However, all these algorithms were derived under the assumption that a sub-band or a subcarrier can be used by one user only. By contrast, our proposed USSCH algorithm allows multiple constant-rate users to activate the same subcarrier. More specifically, each subcarrier can be activated by different users during different hopping intervals, while it is desirable to ensure that the average frequency-domain (FD) separation of the subcarriers activated by the same user is sufficiently large, in order to experience uncorrelated fading, and hence a high diversity gain. Moreover, the number of users activating each subcarrier should ideally be similar so as to ensure that the MUI encountered at each subcarrier becomes similar, hence eliminating the MUI 'peaks' across the system bandwidth.

In order to fully exploit the potential benefits of the USSCH pattern, the employment of forward error correction (FEC) schemes is necessary. In this paper, turbo trelliscoded modulation (TTCM) $[29,30]$ is considered. However, other FEC schemes are also applicable. It will be shown in our forthcoming discourse that with the aid of the advocated USSCH pattern, the MUI experienced by the TTCM-aided SDMA-OFDM system can be effectively suppressed, resulting in a significant performance improvement. In the investigations conducted, the proposed USSCH-aided SDMA-OFDM system was capable of outperforming a range of SDMA-OFDM systems considered, including the conventional SDMA-OFDM system [10] dispensing with the employment of frequency-hopping techniques. For example, at an $E_{b} / N_{0}$ value of $12 \mathrm{~dB}$, the proposed USSCH/SDMA-OFDM system reduced the bit error ratio (BER) by about three orders of magnitude in comparison to the conventional SDMA-OFDM system, while maintaining a similar computational complexity.

\section{System model}

\subsection{System overview}

Figure 1 shows the frequency resource allocation strategy of conventional SDMA-OFDM [10], SFH/SDMA-OFDM and $\mathrm{SSCH} / \mathrm{SDMA}-\mathrm{OFDM}$ systems. As illustrated in Fig. 1, in conventional SDMA-OFDM systems, which do not employ any hopping techniques, the total available system bandwidth $W$ is partitioned into a total of $Q_{c}=W / W_{s c}$ subcarriers, where $W_{s c}$ is the subcarrier bandwidth, and each user activates all the $Q_{c}$ subcarriers for communication. By contrast, in SFH/SDMA-OFDM systems $W$ is divided into a number of sub-bands denoted by $q=1, \ldots, Q_{f}$, each of which consists of $K$ number of subcarriers, as seen in Fig. 1. Explicitly, we have $Q_{c}=K \cdot Q_{f}$. More specifically, during a SFH dwell time $T_{h}$, each of the

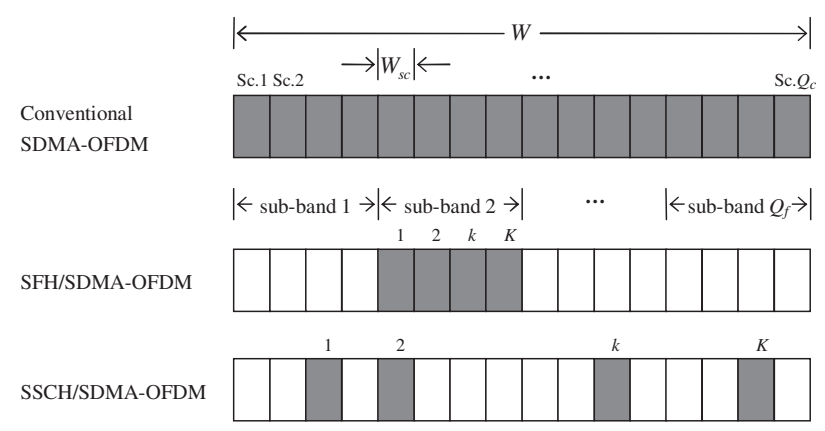

Fig. 1 Comparison between conventional SDMA-OFDM, SFH/ SDMA-OFDM and SSCHISDMA-OFDM in the context of frequency resource allocation for a single user

Each hollow block in the figure represents an unused subcarrier, while those in grey denote the activated subcarriers of the user. In this example, random hopping patterns are used for both the SFH and the $\mathrm{SSCH}$-aided systems

simultaneous users supported is assigned one of the $Q_{f}$ subbands, which is associated with a carrier frequency $f_{q}$ used for carrying the OFDM signal to be transmitted in this subband. The activation of a sub-band is controlled by a frequency synthesiser according to either a pseudorandom or a deterministic user-specific hopping pattern set used. In contrast to fast-frequency-hopping (FFH) systems, where the symbol duration $T_{S}$ obeys $\beta_{F}=T_{S} / T_{h}>1$, in $\mathrm{SFH}$ systems we have $\beta_{S}=T_{h} / T_{S}>1$, where both $\beta_{F}$ and $\beta_{S}$ are integers, while $T_{h}$ is the hopping dwell period. Using SFH makes it feasible to use coherent demodulation at the receiver side, since the hopping rate is slower than the data rate.

In comparison to SFH/SDMA-OFDM systems, where sub-band based SFH is employed, in the proposed $\mathrm{SSCH} /$ SDMA-OFDM system a subcarrier-based SFH technique is invoked. Similar to conventional SDMA-OFDM systems, in SSCH systems we also have a total of $Q_{c}=W / W_{s c}$ subcarriers. However, each user activates only $K$ of the $Q_{c}$ available subcarriers, where $0<K<Q_{c}$. While a SFH user exploits all the $K$ subcarriers of a SFH sub-band during the hopping dwell time of $T_{h}$, a SSCH user employing the same number of $K$ subcarriers can potentially select any $K$ of the $Q_{c}$ available subcarriers without decreasing the throughput, as seen in Fig. 1. Furthermore, by assigning a different number of subcarriers to different users, a flexible multi-rate system can be created, which is capable of satisfying the users' QoS profiles. For the sake of simplicity, in this paper we assume that each user has the same constant bit rate.

\subsection{Transceiver architecture}

The schematic of the TTCM-assisted SSCH/SDMAOFDM system is portrayed in Fig. 2. As seen in Fig. 2, the TTCM-coded symbols are first S/P-converted and forwarded to the SSCH mapper. More specifically, the $K$ number of information symbols of a user are mapped to $K$ out of $Q_{c} \mathrm{SSCH}$ subcarriers, where the activation strategy of the set of $K$ subcarriers depends on the specific SSCH algorithm used, as will be discussed in more depth in Section 2.3. An ON-OFF type signalling scheme may be invoked, where the activated and deactivated status of specific subcarriers represent the ON and OFF states, respectively. Then, the total number of $Q_{c}$ subcarriers can be processed by a $Q_{c}$-point inverse fast Fourier transform (IFFT). Since the deactivated subcarriers deliver no information, the transmit power of each SSCH user is the same as that of a SFH user, provided that the same $K$ number of subcarriers are activated by both of them. 


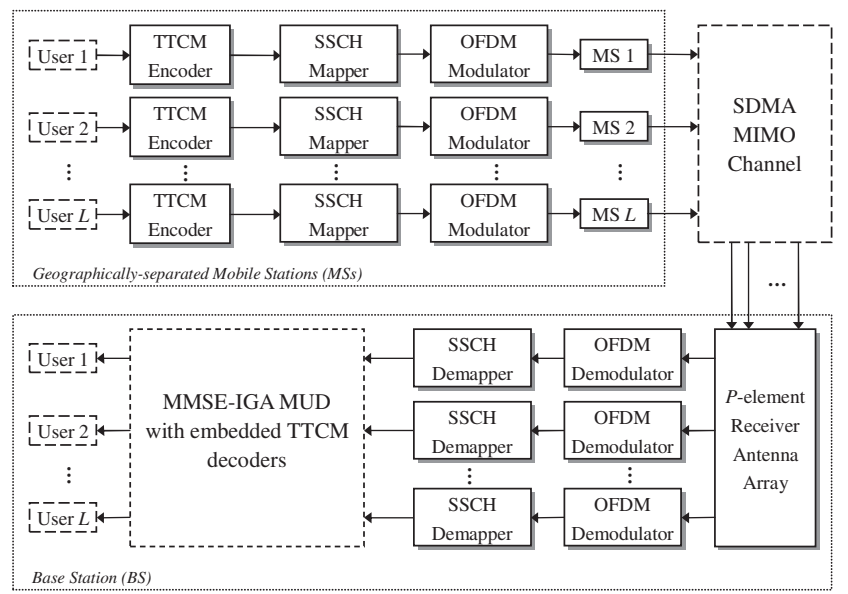

Fig. 2 Schematic of a TTCM-assisted multiuser SSCH-aided SDMA-OFDM uplink system

A modified version of the MMSE-IGA MUD scheme of [31] was employed

Following the IFFT-based OFDM modulation, the OFDM symbols will be transmitted by the mobile station (MS) via the SDMA MIMO channel to be characterised in Section 2.4, using a specific carrier frequency $f_{c}$.

At the BS, a total of $P$ receiver antenna elements and an appropriate MUD are employed. First fast Fourier transform (FFT) based OFDM demodulation is invoked, followed by the $\mathrm{SSCH}$ demapping. Given the knowledge of the SSCH pattern, the BS subcarrier controller simultaneously selects the active subcarriers at the $P$ receivers and the resultant composite multiuser output signal is forwarded to the MUD. Here a modified version of the minimum mean square error (MMSE) aided iterative genetic algorithm (IGA) MUD of [31] is employed, which amalgamates the TTCM decoding process and the MUD operation in an iterative manner. More details about the MUD will be provided in Section 2.5. Finally, the individual users' signals are regenerated.

\subsection{Subcarrier hopping strategy design}

The SSCH pattern decides upon the choice of the subcarriers to which the different users' signals are mapped, and thus has a direct impact on the amount of MUI inflicted. On one hand, for the sake of combating the MUI, a meritorious SSCH pattern should ensure that each user's high-MUI subcarriers are dispersed across the FD, rather than being concentrated in the FD. On the other hand, for the sake of combating FD fading, the subcarriers activated by the same user should be uniformly distributed across the entire bandwidth, rather than being consecutively mapped to a small fraction of the entire bandwidth, so that frequency diversity can be efficiently exploited and hence the detrimental effects of deep FD fades can be mitigated. Both of these requirements result in a more random distribution of the originally bursty FD errors incurred either by a high MUI or a deep FD fade, thus enhancing the chances of the channel decoder to correct the residual errors.

In this paper, two types of hopping patterns are considered. We refer to the first scheme as the random $\mathrm{SSCH}$ (RSSCH) pattern, where each user independently selects any $K$ out of the total of $Q_{c}$ available subcarriers during the period of $T_{h}$. In RSSCH-based systems, the subcarriers activated by a high number of users will suffer an excessive MUI, resulting in an increased average BER. By contrast, in the second SSCH strategy considered, all users' hopping patterns are jointly designed so that the

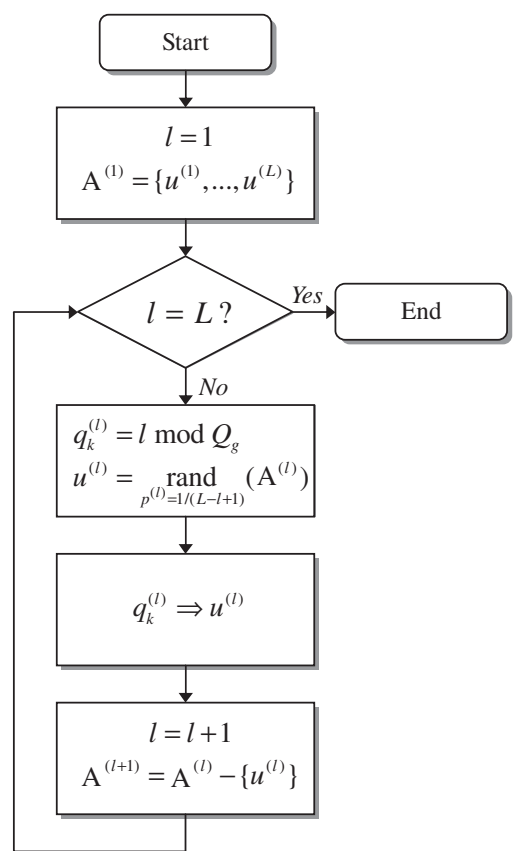

Fig. 3 Flowchart of the USSCH pattern assignment algorithm invoked in the kth subcarrier group

number of interfering users (and also the MUI) at each subcarrier is as similar as possible and that both requirements mentioned above are satisfied. We refer to this strategy as the uniform SSCH (USSCH) scheme.

In the proposed USSCH scheme, the $Q_{c}$ available subcarriers are first partitioned into $K$ consecutive subcarrier groups, each of which has $Q_{g}=Q_{c} / K$ number of subcarriers. Then an $L$-iteration USSCH pattern assignment algorithm is invoked for each of the $K$ groups, namely from the first to the $K$ th group, for the sake of generating a specific length- $K$ pattern set for each of the $L$ users. Figure 3 illustrates the proposed algorithm invoked in the $k$ th $(k=1, \ldots, k)$ subcarrier group for assigning the $Q_{g}$ subcarriers to the $L$ users. More precisely, initially a remaining user set $\boldsymbol{A}^{(1)}$ containing all the $L$ users is created, as shown in Fig. 3. During the $l$ th $(l=1, \ldots, L)$ iteration, a subcarrier index $q_{k}^{(l)}$ is generated by calculating $l \bmod Q_{g}$, where $\bmod$ represents the modulo operation. A user $u^{(l)}$ is then randomly selected from $\boldsymbol{A}^{(l)}$ based on the uniform probability of

$$
p^{(l)}=\frac{1}{L-l+1}
$$

and the $\left(q_{k}^{(l)}\right)$ th subcarrier within this specific subcarrier group will be assigned to user $u^{(l)}$. The remaining user set $\boldsymbol{A}^{(l)}$ is then updated by removing user $u^{(l)}$ from it, resulting in $\boldsymbol{A}^{(l+1)}$, which contains the remaining $(L-l)$ number of users. Then the subcarrier assignment process proceeds to the next, i.e. to the $(l+1)$ th iteration, allocating the next subcarrier to the next randomly selected user, as seen in Fig. 3. This iterative subcarrier to user assignment process continues, until the $L$ th iteration is completed. By this time, each of the $L$ users has been assigned a single subcarrier of the $k$ th subcarrier group. Hence, a vector $\boldsymbol{q}_{k}=\left[q_{k}^{(1)}\right.$, $\left.q_{k}^{(2)}, \ldots, q_{k}^{(L)}\right]$ is generated, where $q_{k}^{(l)}(l=1, \ldots, L)$ constitutes the $k$ th subcarrier of the $l$ th user's length- $K$ USSCH pattern set. (Recall that each user activates $K$ subcarriers for transmission and thus has to be assigned a length- $K$ USSCH pattern set.) Then the vector-generating routine described above is invoked for the next, i.e. for the $(k+1)$ th 
subcarrier group. When all the $K$ subcarrier groups have been processed, a set containing $K$ number of length- $L$ vectors $\boldsymbol{q}_{k}(k=1, \ldots, K)$ has been generated, explicitly indicating, which specific subcarrier of group $k$ has been assigned to which of the $L$ users. Viewing this subcarrier allocation from the users' perspective, the lth user's specific length- $K$ USSCH pattern set $q^{(l)}$ is created by choosing that specific subcarrier corresponding to the $l$ th element of each of the $K$ vectors, which has been assigned to user $l$, resulting in $q^{(l)}=\left[q_{1}^{(l)}, q_{2}^{(l)}, \ldots, q_{K}^{(l)}\right], l \in\{1, \ldots, L\}$.

Note that the above subcarrier group based algorithm employs the full permutation theory [32]. More explicitly, each of the $K$ number of length- $L$ vectors $\boldsymbol{q}_{k}(k=1, \ldots, K)$ associated with the $k$ th subcarrier group is an element of the $L$-order full permutation set [32], and is generated at a constant probability of

$$
\begin{aligned}
p_{L} & =\prod_{l=1}^{L} p^{(l)} \\
& =\frac{1}{L} \cdot \frac{1}{L-1} \cdots \cdot \frac{1}{2} \cdot 1 \\
& =\frac{1}{L !}
\end{aligned}
$$

where $(\cdot)$ ! represents the factorial operation. More explicitly, given a total number of $L$ users, the associated $L$-order full permutation set can be expressed as

$$
\boldsymbol{Q}_{L}=\left\{\boldsymbol{q}_{1}, \boldsymbol{q}_{2}, \ldots, \boldsymbol{q}_{L !}\right\}
$$

where the element vectors are given by $\boldsymbol{q}_{k}=\left[q_{k}^{(1)}, q_{k}^{(2)}\right.$, $\left.\ldots, q_{k}^{(L)}\right](k=1, \ldots, L !)$. Thus, the employment of the USSCH pattern assignment algorithm of Fig. 3 is conceptually equivalent to invoking the algorithm, which randomly selects one element vector from the set $\boldsymbol{Q}_{L}$ described by (3) for each of the $K$ subcarrier groups, based on the probability given by (2).

According to the proposed USSCH pattern assignment algorithm of Fig. 3, when we have $L>Q_{g}$ and $L$ mod $Q_{g} \neq 0$, in each of the $K$ subcarrier groups, there will be $\left(L \bmod Q_{g}\right)$ number of subcarriers that have to be activated by one more user than the other subcarriers in the same group. Thus, a higher MUI is expected at these subcarriers. However, since the users that are assigned to the higherMUI subcarriers are randomly selected, it is unlikely for these more-MUI contaminated subcarriers of different subcarrier groups to be always assigned to the same user. In other words, from each user's point of view, among its total $K$ activated subcarriers, the specific subcarriers that encounter a higher MUI can be uniformly dispersed across the entire system bandwidth. Furthermore, since in USSCH/SDMA-OFDM systems each user's activated subcarriers are uniformly distributed over the entire bandwidth, frequency diversity can be efficiently exploited. Thus, both requirements mentioned at the beginning of this section are satisfied by employing the proposed USSCH pattern assignment algorithm.

However, if we have $L$ mod $Q_{g}=0$, each subcarrier is activated by the same number of $L / Q_{g}$ users. Moreover, when we have $L<Q_{g}$, the USSCH system is actually MUIfree, since each subcarrier is assigned to at most one user. In both of these two scenarios, the MUI encountered in the USSCH/SDMA-OFDM system is identical at all the activated subcarriers, since each subcarrier hosts the same number of users. However, in such situations the performance of the conventional SDMA-OFDM system can still be improved with the aid of the USSCH scheme of Fig. 3, owing to the latter arrangement's ability to exploit frequency diversity.

Note that SFH/SDMA-OFDM systems can also benefit from the proposed USSCH pattern assignment algorithm, resulting in uniform SFH (USFH) aided SDMA-OFDM systems, which are capable of outperforming the conventional SDMA-OFDM systems. In USFH/SDMA-OFDM systems, sub-bands rather than subcarriers are jointly assigned to different users, following a philosophy similar to that of Fig. 3. However, given the same system bandwidth and the same total system throughput, the USFH/SDMA-OFDM system is unable to outperform its USSCH-aided counterpart. This is because in USFH-aided systems a sub-band (which accommodates a number of subcarriers and may be viewed as an 'entire' bandwidth for a 'reduced-size' conventional SDMA-OFDM system) is still more vulnerable both to high MUI and to deep fades than a subcarrier of USSCH-aided systems. This will be corroborated by our simulation results to be shown in Section 3.

It is also worth pointing out that the USFH/USSCH patterns can be acquired by offline precomputation, since their choice is not based on any channel knowledge. Furthermore, the patterns can be reused, provided that the reuse time interval is sufficiently long, so that the frequency diversity can be sufficiently exploited. The reuse interval is defined by

$$
T_{r}=\mu T_{h}
$$

where the reuse factor $\mu$ is a positive integer. More explicitly, during the offline pattern precomputation, $\mu$ number of USFH/USSCH patterns are generated for each of the $L$ users, each of which is associated with one of the $\mu$ number of FH dwell time periods during the time interval of $T_{r}$. These USFH/USSCH pattern sets can then be reused during every reuse interval $T_{r}$, implying that the real-time signalling of the USFH/USSCH patterns from the transmitters to the receivers is unnecessary. This imposes a significantly lower computational complexity than that required by other adaptive algorithms exploiting the realtime channel knowledge.

\subsection{SSCH/SDMA-OFDM uplink MIMO channel}

In SDMA-OFDM uplink systems [10], each of the $L$ simultaneous mobile users employs a single transmit antenna at the MS, while the BS receiver exploits $P$ antenna elements. In the context of the proposed SSCH/SDMAOFDM system, at the $q$ th activated subcarrier of the $n$th OFDM symbol received by the $P$-element receiver antenna array, we have the received complex signal vector $\boldsymbol{x}[n, q]$, which is constituted by the superposition of the independently faded signals associated with the $L_{q}$ users and contaminated by additive white gaussian noise (AWGN), where $L_{q}\left(1 \leq L_{q} \leq L\right)$ denotes the number of users activating the $q$ th subcarrier, expressed as

$$
\boldsymbol{x}_{q}=\boldsymbol{H}_{q} \boldsymbol{s}_{q}+\boldsymbol{n}_{q}
$$

where the $(P \times 1)$-dimensional received signal vector $\boldsymbol{x}_{q}$, the $\left(L_{q} \times 1\right)$-dimensional $L_{q}$ users' transmitted signal vector $\boldsymbol{s}_{q}$ and the $(P \times 1)$-dimensional noise signal vector $\boldsymbol{n}_{q}$ are given by

$$
\begin{aligned}
\boldsymbol{x}_{q} & =\left(x_{1, q}, x_{2, q}, \ldots, x_{P, q}\right)^{T} \\
\boldsymbol{s}_{q} & =\left(s_{q}^{(1)}, s_{q}^{(2)}, \ldots, s_{q}^{\left(L_{q}\right)}\right)^{T} \\
\boldsymbol{n}_{q} & =\left(n_{1, q}, n_{2, q}, \ldots, n_{P, q}\right)^{T}
\end{aligned}
$$


Here we have omitted the indices $[n, k]$ of each vector for the sake of notational convenience. The associated $\left(P \times L_{q}\right)$ dimensional matrix $\boldsymbol{H}_{q}$ of (5), which contains the frequency-domain channel transfer functions (FD-CHTFs) of the $L_{q}$ users, is given by

$$
\boldsymbol{H}_{q}=\left(\boldsymbol{H}_{q}^{(1)}, \boldsymbol{H}_{q}^{(2)}, \ldots, \boldsymbol{H}_{q}^{\left(L_{q}\right)}\right)
$$

where $\boldsymbol{H}_{q}^{(l)}\left(l=1, \ldots, L_{q}\right)$ is the vector of the FD-CHTFs associated with the transmission paths from the $l$ th user's transmit antenna to each element of the $P$-element receiver antenna array, which is expressed as

$$
\boldsymbol{H}_{q}^{(l)}=\left(H_{1, q}^{(l)}, H_{2, q}^{(l)}, \ldots, H_{P, q}^{(l)}\right)^{T}, \quad l=\left\{1, \ldots, L_{q}\right\}
$$

The FD-CHTFs $H_{p, q}^{(l)}$ associated with the different receivers or users are assumed to be independent.

\subsection{Multiuser detection}

As mentioned earlier, MUD schemes are invoked at the receiver of the SDMA-OFDM system for the sake of detecting the received signals of different users. Recently, a novel MMSE-assisted iterative genetic algorithm (IGA) based (MMSE-IGA) MUD [31] has been proposed for employment in conventional SDMA-OFDM systems, which is capable of achieving a similar performance to that attained by the optimum maximum likelihood (ML) MUD $[10,12,13,33]$ at a significantly lower complexity, especially in high-throughput scenarios. In this paper, we modify the MMSE-IGA MUD of [31] so that it can be employed in the proposed SSCH-aided SDMA-OFDM system.

More specifically, at the $q$ th active subcarrier, the initial estimated signal vector $\hat{\boldsymbol{s}}_{\mathrm{MMSE}_{q}} \in \mathbb{C}^{L_{q} \times 1}$ generated from the transmitted signal $\boldsymbol{s}_{q}$ of the $L_{q}\left(1 \leq L_{q} \leq L\right)$ simultaneous users that activate this specific subcarrier, is obtained by linearly combining the received signal vector $\boldsymbol{x}_{q}$ with the aid of the MMSE MUD's array weight matrix, as follows

$$
\hat{\boldsymbol{s}}_{\mathrm{MMSE}_{q}}=\boldsymbol{W}_{\mathrm{MMSE}_{q}}^{H} \boldsymbol{x}_{q}
$$

where the superscript $(\cdot)^{H}$ denotes the Hermitian transpose, and the MMSE-based weight matrix $\boldsymbol{W}_{\mathrm{MMSE}_{q}} \in \mathbb{C}^{P \times L_{q}}$ is given by [10]

$$
\boldsymbol{W}_{\mathrm{MMSE}_{q}}=\left(\boldsymbol{H}_{q} \boldsymbol{H}_{q}^{H}+\sigma_{n}^{2} \boldsymbol{I}\right)^{-1} \boldsymbol{H}_{q}
$$

while $\boldsymbol{I}$ is the identity matrix and $\sigma_{n}^{2}$ is the AWGN noise variance. Once the MMSE-based detection is completed, the resultant output can be forwarded to the concatenated IGA MUD [31] for the second-stage iterative detection. More precisely, the IGA MUD evaluates a decision metric associated with the $P$ receivers, which is derived from the optimum ML-based decision metric [10], in order to detect the estimated transmitted symbol vector $\hat{\boldsymbol{s}}_{\mathrm{GA}_{q}}$. The decision metric required for the $p$ th receiver antenna can be derived from that of [31] as

$$
\Omega_{p, q}(\boldsymbol{s})=\left|x_{p, q}-\boldsymbol{H}_{p, q} \boldsymbol{s}\right|^{2}
$$

and $x_{p, q}$ is the received symbol at the $q$ th active subcarrier of the $p$ th receiver, while $\boldsymbol{H}_{p, q}$ is the $p$ th row of the $\left(P \times L_{q}\right)$ dimensional channel transfer function matrix $\boldsymbol{H}_{q}$ related to the same subcarrier. Therefore, the decision rule for the optimum multiuser detector associated with the $p$ th antenna is to choose that specific length- $L_{q}$ symbol vector $\boldsymbol{s}$, which minimises the metric given in (13). Since there are $P$ number of receivers, the combined joint metric is invoked [31]

$$
\Omega_{q}(\boldsymbol{s})=\sum_{p=1}^{P} \Omega_{p, q}(\boldsymbol{s})
$$

Hence, the joint decision rule is to find that specific estimated transmitted $L_{q}$-symbol vector $\hat{\boldsymbol{s}}_{\mathrm{GA}_{q}}$, which minimises $\Omega_{q}(s)$ in (14) for the $q$ th active subcarrier considered, which is formulated as

$$
\hat{\boldsymbol{s}}_{\mathrm{GA}_{q}}=\arg \left\{\min _{\boldsymbol{s}}\left[\Omega_{q}(\boldsymbol{s})\right]\right\}
$$

For space economy, we omit the details of the IGA-based detection process, which can be found in [31].

\section{Simulation results}

In this Section, we characterise the achievable performance of the proposed TTCM-assisted and USSCH-aided SDMA-OFDM system using the MMSE-IGA MUD of [31]. Its performance is compared to that of its counterparts employing the RSSCH, random SFH (RSFH) and USFH patterns as well as to that of a conventional SDMA-OFDM system [10] dispensing with the employment of any frequency-hopping techniques. It was assumed in all SFHor SSCH-aided schemes that the BS has a perfect knowledge of the hopping patterns. The simulation results were obtained using a 4QAM scheme communicating over the three-path short wireless asynchronous transfer mode (SWATM) CIR given on page 78 of [10], assuming that the channels' transfer functions are perfectly known. Each of the paths experiences independent Rayleigh fading

\begin{tabular}{|c|c|c|}
\hline \multirow[t]{8}{*}{ TTCM parameters } & Modern & 4QAM \\
\hline & Code rate & $1 / 2$ \\
\hline & Data bits & 1 \\
\hline & Parity bits & 2 \\
\hline & Code memory & 3 \\
\hline & Iterations & 4 \\
\hline & Codeword length & 1024 \\
\hline & $\begin{array}{l}\text { Octal generator } \\
\text { polynomial }\end{array}$ & (13 6) \\
\hline \multirow[t]{10}{*}{ GA parameters } & $\begin{array}{l}\text { Population } \\
\text { initialisation method }\end{array}$ & MMSE \\
\hline & $\begin{array}{l}\text { Mating pool creation } \\
\text { strategy }\end{array}$ & Pareto-optimality [34] \\
\hline & Selection method & Fitness-proportionate \\
\hline & Crossover & Uniform crossover \\
\hline & Mutation & BOM [31] \\
\hline & Elitism & Enabled \\
\hline & Incest prevention & Enabled \\
\hline & Population size & 20 \\
\hline & Generations & 5 \\
\hline & Mutation probability & 0.1 \\
\hline \multirow{6}{*}{ Channel parameters } & CIRs & SWATM [10] \\
\hline & Paths & 3 \\
\hline & Maximum path delay & $48.8 \mathrm{~ns}$ \\
\hline & $\begin{array}{l}\text { Normalised Doppler } \\
\text { frequency }\end{array}$ & $1.235 \times 10^{-5}$ \\
\hline & Subarriers & $128 / 512$ \\
\hline & Cyclic prefix & 16 \\
\hline
\end{tabular}
having the same normalised Doppler frequencies of $f_{d}^{\prime}=1.235 \times 10^{-5}$. For the reader's convenience, the simulation parameters are summarised in Table 1. For more details on the GA's configuration, the interested reader is referred to $[3,31]$.

Table 1: Basic simulation parameters used in Section 3 
Recall that in SFH/SSCH aided SDMA-OFDM systems the number of activated subcarriers $K<Q_{c}$ is lower than that of the conventional SDMA-OFDM system [10], where all the users employ all the $Q_{c}$ number of available subcarriers for communication. For the sake of fair comparisons, the total system bandwidth was fixed for all systems and the number of users $L$ supported by the various $\mathrm{SFH} / \mathrm{SSCH}$ aided systems was increased, so that the same total system throughout of $B_{T}$ was maintained during an OFDM symbol, which is calculated by

$$
B_{T}=K \cdot L \cdot B P S \text { [bits/OFDM symbol] }
$$

where $B P S$ represents the number of bits per symbol per subcarrier. When $4 \mathrm{QAM}$ is employed, we have $B P S=2$. For the reader's convenience, the notations used in the figures of this Section are summarised in Table 2.

Figure 4 shows our performance comparison between the conventional SDMA-OFDM and the RSFH, USFH, RSSCH and USSCH aided SDMA-OFDM systems employing either the MMSE MUD [10] or the modified version of the MMSE-IGA MUD [31]. We also included the performance curve of the MUI-free single-user scheme employing the optimum ML detector as a reference, which employed $Q_{c}=512$ subcarriers. TTCM and $P=4$ receiver antenna elements were employed by all systems. The

Table 2: Notations used in the figures of simulation results presented in Section 3

\begin{tabular}{ll}
\hline Notation & Description \\
\hline$K$ & Number of subcarriers employed by each user \\
$L$ & Number of users \\
$P$ & Number of receiver antenna elements \\
$Q_{f}$ & Number of available sub-bands in SFH systems \\
$Q_{e}$ & Number of available subcarriers in SSCH systems \\
$B_{T}$ & Total throughout per OFDM symbol of the $L$-user system \\
RSFG & Random-SFH systems \\
USFH & Uniform-SFH systems \\
RSSCH & Random-SSCH systems \\
USSCH & Uniform-SSCH systems \\
\hline
\end{tabular}

total system throughput was maintained at $B_{T}=6144$ bits per OFDM symbol, except for the single-user reference scheme.

It is observed from Fig. 4 that the conventional SDMAOFDM system resulted in a residual error floor in this highthroughput scenario, which is associated with a high MUI. The RSFH and RSSCH aided systems performed even worse. This is because when the random $\mathrm{SFH} / \mathrm{SSCH}$ strategy is invoked, some subcarriers may be assigned to an excessive number of users, resulting in an extremely high MUI, which contaminates all users' signals delivered by these subcarriers and dominates the average BER performance. However, the situation was significantly improved, when the proposed uniform subcarrier assignment algorithm of Fig. 3 was employed. More specifically, both the USFH-aided SDMA-OFDM systems reduced the error

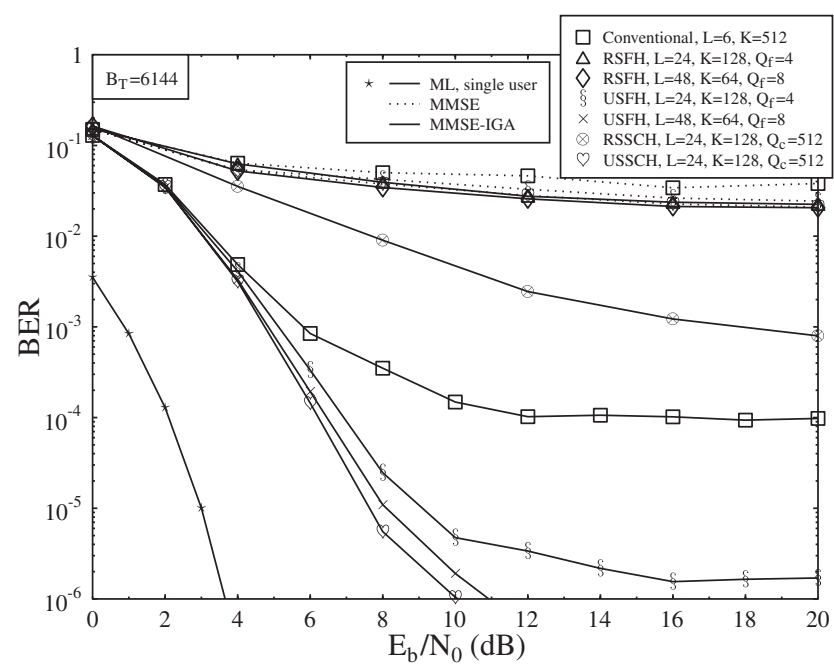

Fig. 4 BER against $E_{b} / N_{0}$ performance of the conventional SDMA-OFDM and RSFH/USFH/RSSCH/USSCH aided SDMA-OFDM systems employing either the MMSE MUD or the MMSE-IGA MUD, where L number of users were supported with the aid of $P=4$ receiver antenna elements

The scenario was associated with a total system throughput of $B_{T}=6144$ bits. All schemes employed TTCM. The basic simulation parameters and the notations used in the figure are summarised in Tables 1 and 2, respectively

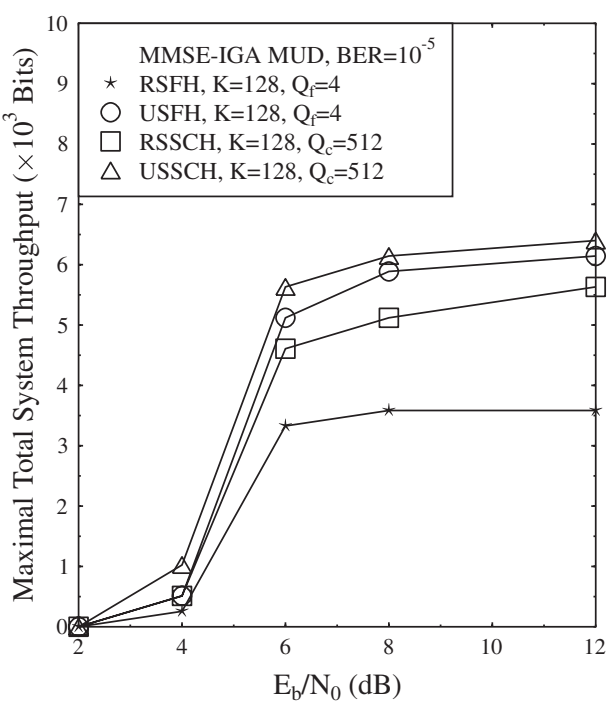

Fig. 5 Maximal total system throughput against $E_{b} / N_{0}$ performance of the RSFH/USFH/RSSCH/USSCH aided SDMA-OFDM systems employing the MMSE-IGA MUD, where $P=4$ receiver antenna elements were used

All schemes employed TTCM. The basic simulation parameters and the notations used in the figure are summarised in Tables 1 and 2, respectively 
floors incurred by their conventional and RSFH/RSSCHaided counterparts, while the USSCH-aided SDMAOFDM system performed even better, validating our arguments outlined in Section 2.3. Furthermore, Fig. 4 also demonstrates the superior performance of the MMSE-IGA MUD over that of the MMSE MUD. For example, at an $E_{b} / N_{0}$ value of $12 \mathrm{~dB}$, the MMSE-IGA multiuser detected USSCH/SDMA-OFDM system dramatically reduced the BER floor of the MMSE multiuser detected USSCH/ SDMA-OFDM system by about five orders of magnitude.

Figure 5 shows the maximum total system throughput $B_{T}$ that can be supported by the various $\mathrm{SFH} / \mathrm{SSCH}$-aided TTCM/SDMA-OFDM schemes without exceeding the target BER at different $E_{0} / N_{0}$ values. The number of subcarriers activated by each user was set to $K=128$ for all schemes for the sake of a fair comparison. Specifically, the results attained for the target BER of $10^{-3}$ and $10^{-5}$ are portrayed at the left-hand and right-hand sides of Fig. 5, respectively. It can be seen that in both scenarios the USSCH/SDMA-OFDM system distinguished itself by providing a higher capacity than the other arrangements, owing to the direct benefit of the USSCH pattern assignment strategy of Fig. 3.

From the results of Figs. 4 and 5, the scheme combining the MMSE-IGA MUD and the USSCH pattern assignment strategy was found to constitute the best design option in terms of the attainable performance. Furthermore, when compared to the conventional SDMA-OFDM system, the only additional computational complexity imposed by the USSCH/SDMA-OFDM system arises from the lowcomplexity USSCH algorithm. Moreover, it allows offline precomputation of the patterns, as discussed at the end of Section 2.3. Thus, the desirably high performance of the proposed USSCH/SDMA-OFDM system is not achieved at the cost of a significantly increased computational complexity, when compared to its conventional counterpart.

\section{Conclusions}

From the investigations conducted, we conclude that the proposed USSCH/SDMA-OFDM system is capable of providing the best performance among the various SDMAOFDM systems investigated, when TTCM is employed. Furthermore, the USSCH-aided system exhibits a robust resistance to the strong MUI incurred in high-throughput scenarios, owing to the attractive characteristics of the proposed subcarrier assignment algorithm. Moreover, the superior performance of the proposed USSCH/SDMAOFDM scheme is achieved at a similar computational complexity as that imposed by the conventional SDMAOFDM arrangement.

\section{Acknowledgments}

The work reported in this paper has formed part of the Wireless Enablers 2.2 of the Core 3 Research Programme of the Virtual Centre of Excellence in Mobile and Personal Communications, Mobile VCE, www.mobilevce.com, whose funding support, including that of EPSRC, is gratefully acknowledged. Fully detailed technical reports on this research are available to Industrial Members of Mobile VCE.

\section{References}

1 Steele, R., and Hanzo, L.: 'Mobile radio communications: second and third generation cellular and WATM systems' (IEEE Press, John Wiley \& Sons Ltd., New York, USA, 1999, 2nd edn.)
2 Viterbi, A.J.: 'CDMA: principles of spread spectrum communication' (Addison-Wesley, 1995)

3 Hanzo, L., Yang, L.-L., Kuan, E.-L., and Yen, K.: 'Single- and multicarrier DS-CDMA: multi-user detection, space-time spreading, synchronisation and standards' (IEEE Press, John Wiley \& Sons Ltd, 2003)

4 Ziemer, R.E., and Peterson, R.L.: 'Digital communications and spread spectrum system' (Macmillan Publishing Company, New York, USA, 1985)

5 Einarsson, G.: 'Address assignment for a time-frequency-coded, spread-spectrum system', Bell Syst. Tech. J., 1980, 59, pp. 1241-1255

6 Goodman, D.J., Henry, P.S., and Prabhu, V.K.: 'Frequency-hopped multilevel FSK for mobile radio', Bell Syst. Tech. J., 1980, 59, pp. $1257-1275$

7 Lam, A.W., and Sarwate, D.P.: 'Time-hopping and frequencyhopping multiple-access packet communications', IEEE Trans. Commun. 1990, 38, pp. 875-888

8 Fiebig, U.: 'Iterative interference cancellation for FFH/MFSK MA systems', IEE Proc. Commun., 1996, 143, pp. 380-388

9 Bingham, J.A.C.: 'Multicarrier modulation for data transmission: an idea whose time has come', IEEE Commun. Mag., 1990, 28,

10 pp. 5-14 CDMA for broadband multi-user communications, WLANs and broadcasting' (IEEE Press, John Wiley \& Sons Ltd, 2003)

11 Koffman, I., and Roman, V.: 'Broadband wireless access solutions based on OFDM access in IEEE 802.16', IEEE Commun. Mag., 2002, 40, pp. $96-103$

12 Vandenameele, P., Perre, L.V.D., and Engels, M.: 'Space division multiple access for wireless local area networks' (Kluwer, London, UK, 2001)

13 Verdu, S.: 'Multiuser detection' (Cambridge University Press, 1998)

14 Jang, J., and Lee, K.B.: 'Transmit power adaptation for multiuser OFDM systems', IEEE J. Sel. Areas Commun., 2003, 21, pp. $171-178$

15 Wong, C.Y., Cheng, R.S., Lataief, K.B., and Murch, R.D.: 'Multiuser OFDM with adaptive subcarrier, bit, and power allocation', IEEE J. Selec. Areas Commun., 1999, 17, pp. 1747-1758

16 amd, C.Y.W., Tsui, C.Y., Cheng, R.S., and Lataief, K.B.: 'A real-time sub-carrier allocation scheme for multiple access downlink OFDM transmission'. Proc. 1999 IEEE 50th Vehicular Technology Conf. (VTC '99 Fall), 19-22 September 1999, Vol. 2, pp. $1124-1128$

17 Kivanc, D., Li, G., and Liu, H.: 'Computationally efficient bandwidth allocation and power control for OFDMA', IEEE Trans. Wirel. Commun., 2003, 2, pp. 1150-1158

18 Kivanc, D., and Liu, H.: 'Subcarrier allocation and power control for OFDMA'. Conf. Record of 34th Asilomar Conf. on Signals Systems, and Computers, 29 October-1 November 2000, Vol. 1, pp. $147-151$

19 Kim, I., Lee, H.L., Kim, B., and Lee, Y.H.: 'On the use of linear programming for dynamic subchannel and bit allocation in multiuser OFDM'. Proc. 2001 IEEE Global Telecommunications Conf. (GLOBECOM '01), 25-29 November 2001, Vol. 6, pp. 3648-3652

20 Rhee, W., and Cioffi, J.M.: 'Increase in capacity of multiuser OFDM system using dynamic subchannel allocation'. Proc. 2000 IEEE 51st Vehicular Technology Conf. (VTC '00 Spring), Tokyo Japan, 15-18 May 2000, Vol. 2, pp. 1085-1089

21 Das, S., and Mandyam, G.D.: 'An efficient sub-carrier and rate allocation scheme for M-QAM modulated uplink OFDMA transmission'. Proc. 2003 IEEE 37th Asilomar Conf. on Signals Systems and Computers (ACSSC '03), 9-12 November 2003, Vol. 1, pp. $136-140$

22 Pietrzyk, S., and Janssen, G.J.M.: 'Multiuser subcarrier allocation for QoS provision in the OFDMA systems'. Proc. 2002 IEEE 56th Vehicular Technology Conf. (VTC '02 Fall), 24-28 September 2002, Vol. 2, pp. 1077-1081

23 Hu, Z., Zhu, G., Xia, Y., and Liu, G.: 'Multiuser subcarrier and bit allocation for MIMO-OFDM systems with perfect and partial channel information'. Proc. 2004 IEEE Wireless Communications and Networking Conf. (WCNC '04), 21-25 March 2004, Vol. 2, pp. $1188-1193$

$24 \mathrm{Li}$, Y., and Sollenberger, N.R.: 'Clustered OFDM with channel estimation for high rate wireless data', IEEE Trans. Commun., 2001, 49, pp. 2071-2076

25 Zhou, S., Giannakis, G.B., and Scaglione, A.: 'Long codes for generalized FH-OFDMA through unknown multipath channels', IEEE Trans. Commun., 2001, 49, pp. 721-733

26 Cao, Z., Tureli, U., and Liu, P.: 'Optimum subcarrier assignment for OFDMA uplink'. Conf. Record of the 37th Asilomar Conference on Signals Systems, and Computers, 9-12 November 2003, Vol. 1, pp. $708-712$

27 Kim, Y.H., Kim, K.S., and Ahn, J.Y.: 'Iterative estimation and decoding for an LDPC-coded OFDMA system in uplink environments'. Proc. 2004 IEEE Int. Conf. on Communications (ICC 04), 20-24 June 2004, Vol. 4, pp. 2478-2482

28 Sari, H., Levy, Y., and Karam, G.: 'An analysis of orthogonal frequency-division multiple access'. Proc. 1997 IEEE Global Telecommunications Conf. (GLOBECOM '97), 3-8 November 1997, Vol. 3, pp. 1635-1639

29 Hanzo, L., Liew, T.H., and Yeap, B.L.: 'Turbo coding, turbo equalisation and space-time coding for transmission over fading 
channels' (IEEE Press, John Wiley \& Sons Ltd., New York, USA, 2002)

30 Robertson, P., and Wörz, T.: 'Bandwidth efficient turbo trellis-coded modulation using punctured component codes', IEEE J. Sel. Area Commun., 1998, 16, pp. 206-218

31 Jiang, M., Ng, S.X., and Hanzo, L.: 'Hybrid iterative multi-user detection for channel coded space division multiple access OFDM systems', IEEE Trans. Veh. Tech., 2006, 55, pp. 115-127
32 Lipschutz, S., and Lipson, M.L.: 'Schaums outline of theory and problems of probability' 'Schaum's outline series' (McGraw-Hill, USA, 2000, 2nd edn.)

33 Vandenameele, P., Perre, L.V.D., Engels, M., Gyselinckx, B., and Man, H.D.: 'A Combined OFDM/SDMA approach', IEEE J. Selec. Areas Commun., 2000, 18, pp. 2312-2321

34 Goldberg, D.E.: 'Genetic algorithms in search, optimization, and machine learning' (Addison-Wesley, Reading, MA, 1989) 